P39 LONGITUDINAL CHANGES OF CEREBRAL WHITE MATTER TISSUE MICROSTRUCTURE IN EARLY-ONSET SYSTEMIC LUPUS ERYTHEMATOSUS

${ }^{1}$ Ettore Silvagni, ${ }^{2}$ Francesca Inglese, ${ }^{1}$ Alessandra Bortoluzzi, ${ }^{3}$ Alfredo Revenaz, ${ }^{3}$ Massimo Borrelli, ${ }^{4}$ Margreet Steup-Beekman, ${ }^{4}$ Tom Huizinga, ${ }^{5}$ Jeroen De Bresser, ${ }^{2}$ Itamar Ronen, ${ }^{6}$ Enrico Fainardi, ${ }^{1}$ Marcello Govoni, ${ }^{2}$ Ece Ercan. ${ }^{1}$ Dept. of Medical Sciences, Rheumatology Unit, Ferrara University, Ferrara, Italy; ${ }^{2}$ Dept. of Radiology, C.J. Gorter Center High Field MRI, Leiden University Medical Center (LUMC), Leiden, The Netherlands; ${ }^{3}$ Dept. of Neuroscience and Rehabilitation, Ferrara, Italy; ${ }^{4}$ Dept. of Rheumatology, LUMC, Leiden; ${ }^{5}$ Dept. of Radiology, LUMC, Leiden, The Netherlands; ${ }^{6}$ Dept. of Experimental and Clinical Biomedical Sciences, Florence University, Florence, Italy

\subsection{6/lupus-2020-eurolupus.87}

Background Diffusion tensor imaging (DTI) studies revealed alterations of cerebral white matter (WM) tissue microstructure in patients with established Systemic Lupus Erythematosus (SLE), highlighting longitudinal changes in DTI metrics. The main aim of this study was to evaluate longitudinal variations of DTI metrics in different WM tracts of newly-diagnosed SLE patients.

Methods In a prospective single-centre observational study (2013-2018), patients meeting revised ACR or SLICC classification criteria, aged less than 55, within 24 months from diagnosis, were evaluated with brain MRI (1.5T Philips Achieva) at baseline (T0) and after at least 12 months (FU). DTI data (15 directions, b-value $800 \mathrm{~s} / \mathrm{mm}^{2}$ ) were analysed using ExploreDTI software. Automatic lesion segmentation was performed using Lesion Prediction Algorithm (Matlab16). An in-house developed semi-automated WM tracts segmentation algorithm was used to assess fractional anisotropy (FA), mean $(\mathrm{MD})$, radial $(\mathrm{RD})$, axial diffusivity $(\mathrm{AD})$ values in different normal-appearing WM tracts. Variations in neuroimaging data were analysed by Wilcoxon matched-pairs signed-ranks test.

Results 17 early SLE patients were included. After mean 456.3 (87.1) days, mean(SD) FA values significantly decreased at left corticospinal tract (T0: $0.483(0.032)$; FU: $0.470(0.034)$, $\mathrm{p}=0.0040)$ and posterior limb of left internal capsule $(0.590$
$(0.020)$ vs $0.580(0.024), p=0.0396)$, with increase in $\mathrm{MD}$ $(0.755$ vs $0.770, p=0.0023)$ and $R D$ values $(0.467$ vs 0.482 , $\mathrm{p}=0.0019$ ) (figure 1). Increase in $\mathrm{MD}$ and $\mathrm{RD}$ values was independent of baseline neurologic symptoms, disease activity and comorbidities.

Conclusions Longitudinal decrease in FA and increase in MD start in early phases of SLE course, even in absence of overt NP symptoms, reflecting a compromised WM tissue microstructure.

\section{P40 PROMISING SALIVARY PROTEIN BIOMARKERS IN KOREAN PATIENTS WITH SYSTEMIC LUPUS ERYTHEMATOSUS}

Chang-Hee Suh, Ju-Yang Jung, Wook-Young Baek, In-Ok Son. Dept. of Rheumatology, Ajou University School of Medicine, Suwon, Korea

\subsection{6/lupus-2020-eurolupus.88}

Background Systemic Lupus Erythematosus (SLE) is a systemic autoimmune disease characterized by the production of autoantibodies. We aimed to find salivary protein biomarkers in Korean patients with SLE.

Methods The salivary proteins were subjected to 2-dimensional gel electrophoresis (2-DE). The spots exhibiting $>2$-fold intensity change between SLE and healthy subjects (HSs) were identified by matrix-assisted laser desorption/ionization time-offlight mass spectrometry (MS) analysis. The amounts of candidate proteins in saliva of patients with SLE and rheumatoid arthritis (RA), and HSs were analyzed using western blotting and enzyme-linked immunosorbent assay.

Results The proteomic analysis using 2-DE and MS revealed 10 differentially expressed protein spots, which included immunoglobulin gamma-3 chain C (IGHG3), protein S100, lactoferrin, leukemia-associated protein 7, and 8-oxoguanine DNA glycosylase. The patients with SLE exhibited enhanced salivary IGHG3 $(3.9 \pm 2.15 \mathrm{pg} / \mathrm{mL})$ and lactoferrin $(4.7 \pm$

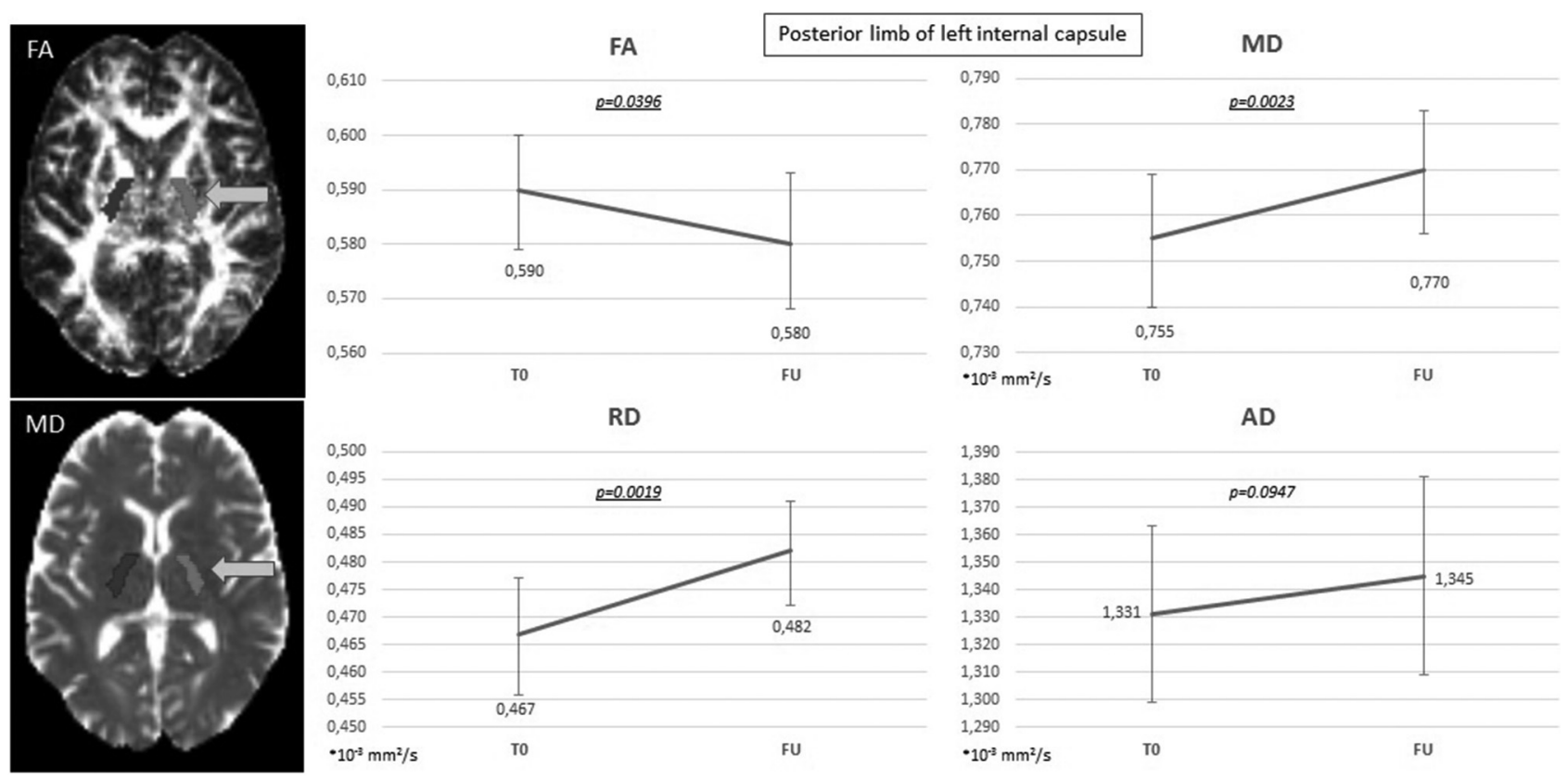

Abstract P39 Figure 1 Axial FA and MD maps with overlying WM tracts (thresholded by the WM probability map), showing posterior limb of left internal capsule (arrows); variation of main DTI parameters (FA, MD, RD, AD) between TO and FU at posterior limb of left internal capsule 